\title{
Dirac operators and manifolds with boundary
}

\author{
Booss-Bavnbek, Bernhelm; Wojciechowski, K.P.
}

\section{Publication date:}

1993

\section{Document Version}

Publisher's PDF, also known as Version of record

Citation for published version (APA):

Booss-Bavnbek, B., \& Wojciechowski, K. P. (1993). Dirac operators and manifolds with boundary. Roskilde Universitet. Tekster fra IMFUFA No. 248 http://milne.ruc.dk/ImfufaTekster/

\section{General rights}

Copyright and moral rights for the publications made accessible in the public portal are retained by the authors and/or other copyright owners and it is a condition of accessing publications that users recognise and abide by the legal requirements associated with these rights.

- Users may download and print one copy of any publication from the public portal for the purpose of private study or research. - You may not further distribute the material or use it for any profit-making activity or commercial gain.

- You may freely distribute the URL identifying the publication in the public portal.

\section{Take down policy}

If you believe that this document breaches copyright please contact rucforsk@kb.dk providing details, and we will remove access to the work immediately and investigate your claim. 


\section{TEKST NR 248}

1993

B. Booß-Bavnbek

K. P. Wojciechowski

\section{DIRAC OPERATORS AND MANIFOLDS WITH BOUNDARY}

\section{TEKSTER fra}

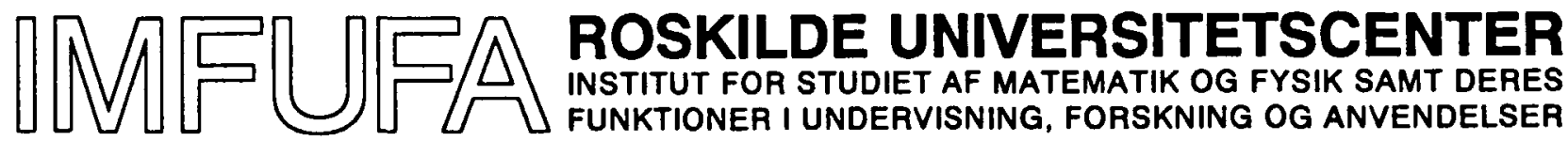


IMFUFA, Roskilde Universitetscenter, Postboks 260, 4000 Roskilde

DIRAC OPERATORS AND MANIFOLDS WITH BOUNDARY

by: B. Booß-Bavnbek and K. P. Wojciechowski

MMUFA tekst nr. 248/93

12 pages

ISSN 0106-6242

\begin{abstract}
.
We deal with various elliptic analogies to the classical Dirac equation; we explain our main analytical tools: invertible extension, Calderón projector, and twisted orthogonality of Cauchy data spaces; we investigate natural spaces of global elliptic boundary value problems for Dirac operators; and we develop an index theory for transmission problems and give additivity and non-additivity theorems for the index and the eta-invariant under cutting and pasting of Dirac operators over partitioned manifolds. The explicit formulas rely on Clifford multiplication with vectors normal to the cutting submanifold.
\end{abstract}




\title{
DIRAC OPERATORS AND MANIFOLDS WITH BOUNDARY •
}

\author{
B. BOOSS-BAVNBEK \\ Institut for matemetik of fyoik \\ Roskilde Univeroity \\ DK.4000 Roskilde, Denmerk \\ e-meil: booss@jane.ruc.dk \\ and \\ K. P. WOJCIECHOWSKI ${ }^{\dagger}$ \\ Dept. Math., IUPUI \\ Indianapolis IN 16208, U.S.A. \\ e-mail: kwojciec(1indyvax.iupui.edu
}

\begin{abstract}
We deal with various elliptic analogies to the classical Dirac equation; we explain our main analytical tools: invertible extension, Calderón projector, and twisted orthogonality of Cauchy data spaces; we investigate natural spaces of global elliptic boundary value problems for Dirac operators; and we develop an index theory for transmission problems and give additivity and non-additivity theorems for the index and the n-invariant under cutting and pasting of Dirac operators over partitioned inanifolds. The explicit formulas rely on Clifford multiplication with vectors normal to the cutting submanifold.
\end{abstract}

Key words: Calderón projector, Clifford modules, Dirac operalor, elliptic boundary problems, eta-invariant, index theory, partitioned manifolds, pseudo-differential Grassmannian, spectral now, surgery

\section{Begin With Clifford Modules}

There are many different concepts of a Dirac operator in global analysis: classical and twisted Dirac operators on spin manifolds; operators of Dirac type with a square with scalar principal symbol; generalized (or compatible) Dirac operators defined by arbitrary (or compatible) connections on bundles of Clifford modules over Riemannian manifolds; full and split (odd-parity) Dirac operators; boundary Dirac operators; etc. The concepts depend on various geometrical features like dimension parity, orientation and chirality, almost complex structure, and suitable boundary. Each definition has its own merits and range of application.

Let $X$ be a compact smooth orienied manifold (with or without boundary) with Riemannian metric $g$. Let $\operatorname{dim} X=n$. Let $S$ be a complex vector bundle over $X$ of Clifford modules; i.e. we have a representation

$$
\text { c : } \mathrm{C} \ell(X) \longrightarrow \operatorname{Hom}(S, S)
$$

- To appeas in R. Delanghe, F. Brackx and H. Serras (eds.), Clifford Algebras and their Applications in Mathematical Phyaics, Proceedings of the Third International Conference (Deinze, Belgium, May 1993), Kluwer Academic Publishers, Dordrecht.

1 Work supported in part by NSF grant no. DMS-9105057. 
with

$$
c(v)^{2}=-\|v\|^{2} \mathrm{Id} s_{x} \quad \text { for } v \in T X_{x} \text { and } x \in X .
$$

Recall that the Clifford bundle $\mathrm{C} \ell(X)$ consists of the Clifford algebras $\mathrm{C} \ell\left(T X_{x}, g_{x}\right)$, $x \in X$, which are associative algebras with unit generated by $T X_{x}$ and subject to the relation $v \cdot w+w \cdot v=-2 g_{x}(v, w)$. We shall call c left Clifford multiplication and occasionally write

$$
\text { c : } C^{\infty}(X ; T X \otimes S) \longrightarrow C^{\infty}(X ; S) .
$$

We may assume that $S$ is equipped with a Hermitian metric which makes Clifford multiplication skew-adjoint, i.e. $c(v)^{\bullet}=-c(v)$ for all $v \in T X_{s}$.

Definition 1 A connection $D: C^{\infty}(X ; S) \longrightarrow C^{\infty}\left(X ; T^{*} X \otimes S\right)$ for $S$ will be called compatible with the Clifford module structure of $S$, if it is Leibnizian, i.e. it satisfies the product rule

$$
\partial_{u}\left\langle s, s^{\prime}\right\rangle=\left\langle D_{v} s, s^{\prime}\right\rangle+\left\langle s, D_{v} s^{\prime}\right\rangle
$$

and if $D c=0$, i.e $D$ is a module derivation with

$$
(D \mathrm{c})(v)(s)=D(\mathrm{c}(v) s)-\mathrm{c}\left(D^{\prime} v\right) s-\mathrm{c}(v)(D s)=0,
$$

where $D^{g}$ denotes the Levi-Civita connection on $X$.

Patching locally constructed spin connections together proves

Theorem 2 (Branson, Gilkey [12]) There exist compatible connections on $S$ which extend the Riemannian connection on $X$ to $S$.

Definition 3 Let $A: C^{\infty}(X ; S) \longrightarrow C^{\infty}(X ; S)$ be a linear differential operator of first order operating on smooth sections of a $C \ell(X)$-module $S$.

(a) We call $A$ an operator of Dirac type, if the principal symbol of its square is defining the Riemannian metric:

$$
\sigma_{\Lambda^{\nu}}(x, \xi)=\sum_{\mu, \nu=1}^{n} g^{\mu \nu}(x) \xi_{\mu} \xi_{\nu} .
$$

(b) We call $A$ a generalized Dirac operator, if it can be written as $A=\mathrm{c} \circ \mathrm{J} \circ D$, where $D$ is a (not necessarily compatible) connection and

$$
J: C^{\infty}\left(X ; T^{*} X \otimes S\right) \cong C^{\infty}(X ; T X \otimes S)
$$

denotes the canonical identification. In terms of a local orthonormal frame $v_{1}, \ldots, v_{n}$ of $T X$ we then have

$$
\left.A s\right|_{x}=\left.\sum_{\nu=1}^{n} c\left(v_{\nu}^{\prime}\right)\left(D_{u_{\nu}} s\right)\right|_{x} .
$$

(c) We call $A$ a (compatible) Dirac operator, if it can be written as $A=c \circ J \circ D$, where $D$ is a compatible connection. 
Note. In this article we deal with compatible Dirac operators. However, most of the arguments remain valid for generalized non-compatible Dirac operators like the Dolbeault complex or, even more general, operators of Dirac type.

Clearly all (total) Dirac operators are elliptic and formally self-adjoint with a Green's formula

$$
\left(A s, s^{\prime}\right)-\left(s, A s^{\prime}\right)=-\int_{Y} G(y)\left\langle\left. s\right|_{Y},\left.s^{\prime}\right|_{Y}\right)_{1}
$$

where $G(y):=c(n)$ denotes Clifford multiplication by the inward unit tangent vector.

For even $n$ the splitting $\mathrm{C} \ell(X)=\mathrm{C} \ell^{+}(X) \oplus \mathrm{C}^{-}(X)$ of the Cliford bundles induces a corresponding splitting of $S=S^{+} \oplus S^{-}$and a chiral decomposition

$$
A=\left(\begin{array}{cc}
0 & A^{-} \\
A^{+} & 0
\end{array}\right) \text {. }
$$

The partial Dirac operators $A^{ \pm}$are especially interesting in index theory since they are also elliptic, but in general not self-adjoint and provide interesting integer-valued invariants as their indices. Like the Cauchy-Riemann operator $\delta=\frac{1}{2}\left(\partial_{x}+i \partial_{y}\right)=$ $\frac{1}{2} e^{i \varphi}\left(\partial_{r}+\frac{i}{r} \partial_{\varphi}\right)$ on the punctured two-disc all partial Dirac operators $A^{+}$can be written in product form

$$
A^{+}=G(u, y)\left(\partial_{u}+B_{u}\right)
$$

close to the boundary, where $u$ denotes the inward oriented normal coordinate. Notice that the Clifford multiplication $G(u, y)$ defines a unitary morphism $\left.S^{+}\right|_{Y} \rightarrow$ $S^{-} \mid Y$ and that $\left\{B_{u}\right\}$ is a family of self-adjoint (total) Dirac operators over $Y$. In the cylindrical case of a product metric close to $Y$ the operators $B_{u}$ and the morphisms $G(u, y)$ are independent of $u$.

\section{Three Analysis Tools}

We shall build our analysis on three basic properties which are not widely known and seem partly overlooked (2.1 and 2.3), partly insufficiently exploited (2.2) in the literature on partial differential equations.

\subsection{INVERTIBLE EXTENSION}

Clifford multiplication by the inward normal vector gives a natural clutching of $S^{+}$ over one copy of $X$ with $S^{-}$over a second copy of $X$ to a smooth bundle $\widetilde{S^{+}}$over the closed double $\tilde{X}$. As observed in [39], the product forms of $A^{+}$and $A^{-}$fit together over the boundary and provide a Dirac operator

$$
\widetilde{A^{+}}:=A^{+} \cup A^{-}: C^{\infty}\left(\tilde{X} ; \widetilde{S^{+}}\right) \longrightarrow C^{\infty}\left(\tilde{X} ; \widetilde{S^{-}}\right) .
$$

Clearly $\left(A^{+} \cup A^{-}\right)^{*}=A^{-} \cup A^{+}$; hence index $\widetilde{A^{+}}=0$. It turns out that $\widetilde{A^{+}}$is invertible with a pseudo-differential elliptic inverse $\left(\widetilde{A^{+}}\right)^{-1}$. Of course $A^{+}$is not invertible and $r^{+}\left(\widetilde{A^{+}}\right)^{-1} e^{+} A^{+} \neq \mathrm{Id}$, where $e^{+}: L^{2}\left(X ; S^{+}\right) \rightarrow L^{2}\left(\tilde{X} ; \widetilde{S^{+}}\right)$denotes the extension by zero operator and $r^{+}: \mathcal{H}^{t}\left(\tilde{X} ; \widetilde{S^{+}}\right) \rightarrow \mathcal{H}^{t}\left(X ; S^{+}\right)$the natural restriction operator for Sobolev spaces, $t$ real. 


\subsection{Calderón Projector}

The next piece is the Calderón projector. It is a pseudo-differential projection onto the Cauchy data spaces (announced in Calderón [14] and proved in Seeley [31] in great generality). We define the Cauchy data spaces

$$
H_{+}\left(A^{+}\right):=\left\{s|Y| s \in C^{\infty}\left(X ; S^{+}\right) \text {and } A^{+} s=0 \text { in } X \mid Y\right\}
$$

and, for real $t$,

$$
H_{+}\left(A^{+}, t\right):=\text { closure of } H_{+}\left(A^{+}\right) \text {in } \mathcal{H}^{t-\frac{1}{5}}\left(\left.S^{+}\right|_{\boldsymbol{Y}}\right) ;
$$

and the null spaces

$$
\operatorname{ker}_{+}\left(A^{+}, t\right):=\left\{s \in \mathcal{H}^{t}\left(X ; S^{+}\right) \mid A^{+} s=0 \text { in } X \backslash Y\right\} .
$$

The null spaces consist of sections which are distributional for negative $t$; which by elliptic regularity are smooth in the interior; and which by a Riesz, operalor argument can be shown to possess a trace $\gamma_{0}(s)$ over the boundary in $\mathcal{H}^{i-\frac{1}{b}}\left(Y ;\left.S^{+}\right|_{Y}\right)$.

First we construct a Poisson type operator

$$
K_{+}:=r^{+}\left(\overline{A^{+}}\right)^{-1}\left(\gamma_{0}^{-}\right)^{\bullet} G: C^{\infty}\left(\left.S^{+}\right|_{Y}\right) \rightarrow C^{\infty}\left(\left.S^{+}\right|_{X \backslash Y}\right) .
$$

It extends to a continuous mapping $\mathcal{H}^{1-\frac{1}{2}}\left(\left.S^{+}\right|_{Y}\right) \rightarrow \operatorname{ker}_{+}\left(A^{+}, t\right)$ which is a bijection, if restricted to $H_{+}\left(A^{+}, t\right)$. Then we obtain the Calderón projector by taking the traces

$$
\mathcal{P}_{+}:=\lim _{u \rightarrow 0_{+}} \gamma_{u} K_{+}
$$

and similarly $\mathcal{P}_{-}:=-\lim _{u \rightarrow 0_{-}} \gamma_{u} K_{+}$. Then $\mathcal{P}_{+}$is a pseudo-differential projection with range $\left(\mathcal{P}_{+}\right)=H_{+}\left(A^{+}\right)$and $\mathcal{P}_{+}+\mathcal{P}_{-}=$Id.

The principal symbol $p_{+}$of the Calderón projector is the projection onto the eigenspaces of the principal symbol $b(y, \zeta)$ of $B_{0}$ corresponding to non-negative eigenvalues. Hence it coincides with the principal symbol of the spectral projection $\mathrm{P}_{\geq}\left(B_{0}\right)$. We call the space of pseudo-differential projections with the same principal symbol $p_{+}$the Grassmannian $\mathrm{Gr}_{p_{+}}$. It has enumerable many connected components; two projections $P_{1}, P_{2}$ belong to the same component, if and only if the virtual codimension

$$
\mathrm{i}\left(P_{2}, P_{1}\right):=\text { index }\left\{P_{2} P_{1}: \text { range } P_{1} \rightarrow \operatorname{range} P_{2}\right\}
$$

of $P_{2}$ in $P_{1}$ vanishes; the higher homotopy groups of each connected component are given by Bott periodicity.

\subsection{Twisted Orthogonality of Cauchy Data Spaces}

It is a nice feature of the Clifford multiplication $G$, first observed in [9], that it describes the orthogonal complement of the Cauchy data space of $A^{+}$by

$$
G^{-1}\left(H_{+}\left(A^{-}\right)\right)=\left(H_{+}\left(A^{+}\right)\right)^{\perp}
$$

and provides a short exact sequence

$$
0 \rightarrow G^{-1}\left(H_{+}\left(\Lambda^{-}, t\right)\right) \hookrightarrow \mathcal{H}^{t}\left(Y ; S^{+} \mid \gamma\right) \stackrel{K_{+}}{\rightarrow} \operatorname{ker}_{+}\left(\Lambda^{+}, t\right) \rightarrow 0 .
$$




\section{Some Basic Results}

To get a closed operator, full regularity of the solutions, and a finite integer-valued index for Dirac operators over manifolds with boundary, we must impose global elliptic boundary conditions.

Definition 4 A pseudo-differential operator $R: C^{\infty}\left(Y ; S^{+} \mid y\right) \rightarrow C^{\infty}(Y ; V)$ of order 0 defines an elliptic boundary condition for $A^{+}$, if range $R^{(t)}$ is closed in $\mathcal{H}^{\imath}(Y ; V)$ and range $(r)=$ range $\left(r p_{+}\right)=$range $\left(p_{+}\right)$. Here $R^{(l)}$ denotes the continuous extension of $R$ to the $t$-th Sobolov space and $r$ the principal symbol of $R$.

Examples 5 Typical examples are the Atiyah-Patodi-Singer boundary condition defined by the spectral projection $\mathrm{P}_{\geq}$of the boundary Dirac operator $B_{0}$; generalized Atiyah-Patodi-Singer boundary conditions defined by projections belonging to the Grassmannian $\mathrm{Gr}_{p_{+}} ;$and local elliptic boundary conditions characterized by the additional condition that the range of $r$ can be written as the lifting of the vector bundle $V$ under the natural projection $T^{*} Y \backslash 0 \rightarrow Y$. For even-dimensional $X$ the Clifford multiplication becomes non-trivial and extludes the existence of local elliptic boundary conditions for $A$ and $A^{+}$(though not for systems); for odd-dimensional $X$ we have natural local elliptic boundary conditions $\Pi_{ \pm}$defined by the orthogonal projection of $\left.S\right|_{Y}$ onto $\left(\left.S\right|_{Y}\right)^{ \pm}$.

Theorem 6 The operator $A_{R}^{+}: \operatorname{dom} A_{R}^{+} \rightarrow L^{2}\left(X ; S^{-}\right)$, which acts like $A^{+}$and is determined by

$$
\operatorname{dom} A_{R}^{+}:=\left\{s \in \mathcal{H}^{1}\left(X_{i} s^{+}\right) \mid R^{(0)}\left(\gamma_{0} s\right)=0\right\},
$$

is a Fredholm operator from $L^{2}\left(X ; S^{+}\right)$to $L^{2}\left(X ; S^{-}\right)$with index $A_{R}^{+}=\mathrm{i}\left(R, \mathcal{P}_{+}\right)$.

Proof A first proof was sketched in Seeley [35]. We show how easy it is in our context: The subspace ker $A_{R}^{+}$of $\mathcal{H}^{1}\left(X ; S^{+}\right)$consists of smooth sections, since $A^{+} s=$ 0 and $R\left(\gamma_{0} s\right)=0$ imply that $h:=\gamma_{0} s$ belongs to the kernel of the boundary integral $R P_{+}: H_{+}\left(A^{+}, 1\right) \rightarrow$ range $R^{(1)}$ which is contained in the kcrnel of the 'fan' $\left(\mathrm{Id}-\mathcal{P}_{+}\right)+\mathcal{P}_{+} R^{*} R \mathcal{P}_{+} ;$the fan is elliptic by the symbol compatibility condition of Definition 4 ; hence $h$ is smooth; hence also $s=K_{+} h$ is smooth. (The concept of an elliptic $f a n$ is due to $M$. Birman and $A$. Solomyak [6]). The argument establishes the isomorphism

$$
\operatorname{ker} A_{R}^{+} \cong \operatorname{ker}\left\{R \mathcal{P}_{+}: H_{+} \longrightarrow \operatorname{range}(R)\right\}
$$

and the finite dimension of the kernel. That $A_{R}^{+}$is a closed $L^{2}$ realization can be deduced from the explicit description of a left parametrix for $A^{+}$by

$$
\left(r^{+}\left(\widetilde{A^{+}}\right)^{-1} e^{+}\right) A^{+}=\mathrm{Id}-K_{+} \gamma_{0} \text {, }
$$

which is a direct consequence of the Calderón construction. Now all is simple: Since we have an explicit description of the adjoint operator

$$
\left(A_{R}^{+}\right)^{*}=A_{G(\mathrm{Id}-R) G \cdot}^{-}
$$

(if $R$ is a projection; otherwise replace $R$ by the orthogonal projection onto the range of $R^{*}$ ) and since the range of $A_{R}^{+}$is closed in $L^{2}$, we also have an explicit description of the cokernel of $A_{R}^{+}$which, by the Clifford rotation of Cauchy data spaces of (8), can be identified with the cokernel of the boundary integral. 
It follows from the topology of $\mathrm{Gr}_{p_{+}}$that for generalized Atiyah-Patodi-Singer boundary conditions index $A_{R}^{+}$vanishes, if and only if the projection $R$ belongs to the same connected component of $\mathrm{Gr}_{p_{+}}$as the Calderón projector $\mathcal{P}_{+}$. More generally, we obtain two explicit versions of the classical Agronovic-Dynin formula (see [1]).

Theorem 7 For two projections $R_{1}$ and $R_{2} \in \mathrm{Gr}_{p_{+}}$we have

$$
\text { index }\left(A_{R_{1}}^{+}\right) \text {-index }\left(A_{R_{2}}^{+}\right)=\mathrm{i}\left(R_{1}, R_{2}\right) \text {; }
$$

and for two local elliptic boundary conditions $R_{j}: C^{\infty}\left(\left.Y_{;} S^{+}\right|_{Y}\right) \rightarrow C^{\infty}\left(Y_{i} V_{j}\right)$, $j=1,2$, we have

$$
\text { index }\left(A_{R_{1}}^{+}\right)-\operatorname{index}\left(A_{R_{2}}^{+}\right)=\operatorname{index}\left\{R_{1} \mathcal{P}_{+} R_{2}: C^{\infty}\left(Y ; V_{2}\right) \rightarrow C^{\infty}\left(Y ; V_{1}\right)\right\} .
$$
[3]:

From (9) we get a generalization of the Atiyah-Patodi-Singer index formula of

Theorem 8 For $R \in \mathrm{Gr}_{\mathrm{p}+}$ we have

$$
\text { index } A_{R}^{+}=\int_{X} \alpha(x)-\frac{1}{2}\left(\eta_{B}(0)+\operatorname{dimker} B\right)+\mathrm{i}\left(R, \mathrm{P}_{\geq}\right) \text {. }
$$

Here $\alpha(x)$ denotes the locally defined index density of $A^{+}$and

$$
\eta_{B}(z):=\sum_{\lambda \in \operatorname{spec} B \backslash\{0\}} \operatorname{sign} \lambda|\lambda|^{-x}=\frac{1}{\Gamma\left(\frac{z+1}{2}\right)} \int_{0}^{\infty} t^{\frac{x-1}{3}} \operatorname{tr}\left(B e^{-t B^{2}}\right) d t
$$

denotes the $\eta$-function of $B$. It is (i) well defined through absolute convergence for $\Re(z)$ large; (ii) it extends to a meromorphic function in the complex plane with isolated simple poles; (iii) its residues are given by a local formula; and (iv) it has a finite value at $z=0$ (see e.g. Gilkey [16]).

Theorem 8 separates the contributions to the index from the whole manifold, from the structure on the boundary, and from the boundary condition in relation to the structure on the boundary. A special feature is that the correction term $\mathrm{i}(R, \mathrm{P} \geq)$ is not a homotopy invariant. It can change, e.g. under smooth deformations of the Riemannian metric, and is therefore a good candidate for more refined geometrical invariants.

To look at the geometrical aspects more closely, it is, as usual when working with Clifford algebras, appropriate to distinguish between the even- and the odddimensional case; and to alternate the focus between the index, the spectral now, and the $\eta$-invariant.

\section{Even Dimension. Index Theory}

Let $M=X_{1} \cup X_{2}$ be an even-dimensional closed partitioned manifold with $\partial X_{1}=$ $\partial X_{2}=X_{1} \cap X_{2}=Y$. To illustrate the twisting of Cauchy data spaces by Clifford multiplication we prove 


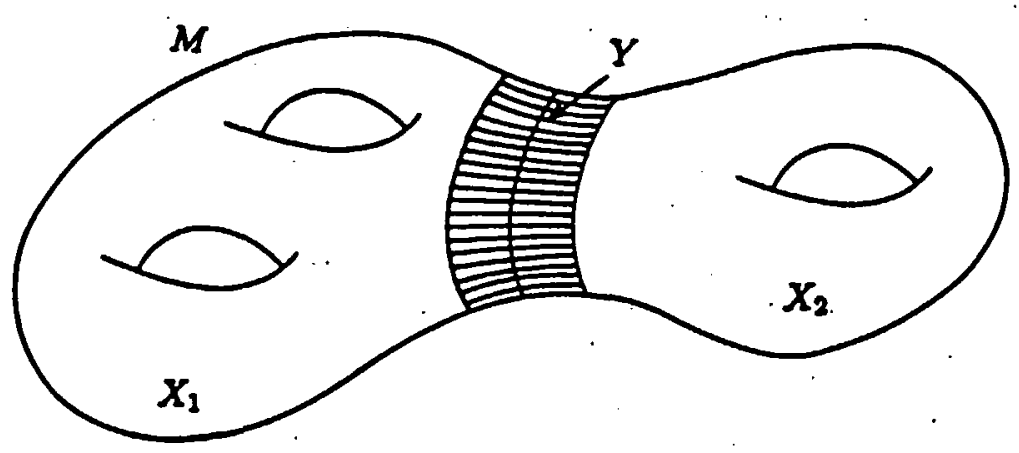

Fis. 1. Partitioned manifold

Theorem $\theta$ (Bojarski's conjecture [7]) Let $A$ be a Dirac operator over $M$, let $A^{j}$ denote its restriction to $X_{j}$, and let $\mathcal{P}\left(A^{j}\right)$ and $H\left(A^{j}\right)$ denote the corresponding Calderón projectors and $L^{2}$ closures of the Cauchy data spaces, $j=1,2$. Then

$$
\text { index } A=\mathrm{i}\left(\mathrm{Id}-\mathcal{P}\left(A^{2}\right), \mathcal{P}\left(A^{1}\right)\right)=\operatorname{index}\left(H\left(A^{1}\right), H\left(A^{2}\right)\right) \text {. }
$$

Note. Notice that Id $-\mathcal{P}\left(A^{2}\right)=G \mathcal{P}\left(A^{1}\right) G^{*}$ by $(8)$ and recall that

$$
\mathbf{i}\left(\mathrm{Id}-\mathcal{P}\left(A^{2}\right), \mathcal{P}\left(A^{1}\right)\right):=\operatorname{index}\left\{\left(\mathrm{Id}-\mathcal{P}\left(A^{2}\right)\right) \mathcal{P}\left(A^{1}\right): H\left(A^{1}\right) \longrightarrow H\left(A^{2}\right)^{\perp}\right\}
$$

and

index $\left(H\left(A^{2}\right), H\left(A^{2}\right)\right):=\operatorname{dim}\left(H\left(A^{2}\right) \cap H\left(A^{2}\right)\right)-\operatorname{dim}\left(L^{2}\left(Y ;\left.S\right|_{Y}\right) /\left(H\left(A^{1}\right)+H\left(A^{2}\right)\right)\right.$.

Note. Closed subspaces for which the two dimensions in the preceding definition are finite are called Fredholm pairs of subspaces.

Proof It follows from the unique continuation property for Dirac operators and Green's formula (5) that $\operatorname{ker} A \cong H\left(A^{2}\right) \cap H\left(A^{2}\right)$. Now we apply the Clifiord rotation formula for Cauchy data spaces (8) to get coker $A \cong \operatorname{ker} A^{*} \cong G\left(H\left(A^{1}\right)^{\perp}\right) \cap$ $G\left(H\left(A^{2}\right)^{\perp}\right)$. The last space is isomorphic to $H\left(A^{1}\right)^{\perp} \cap H\left(A^{2}\right)^{\perp}$ which is the orthog: onal complement of $H\left(A^{1}\right)+H\left(A^{2}\right)$ in $L^{2}\left(Y ;\left.S\right|_{Y}\right)$.

Replacing the Clifford multiplication with an arbitrary unitary automorphism $\Phi$ of $\left.S\right|_{\gamma}$, which is consistent (i.e. $\Phi$ commutes with $p_{+}$), leads us to the general linear conjugation problem, a generalization of the classical Riemann-Hilbert problem: We are looking for couples $\left(s_{1}, s_{2}\right)$ with

$$
A^{j} s_{j}=0 \text { in } X_{j} \backslash Y \text { for } j=1,2 \text { and }\left.s_{2}\right|_{Y}=\Phi\left(\left.s_{1}\right|_{Y}\right) \text {. }
$$

Let index $\operatorname{LCP}(A, \Phi)$ denote the difference between the dimensions of the solution $\because$ spaces of the original problem (12) and of the corresponding adjoint problem. We obtain

Theorem $10([8],[9])$

$$
\operatorname{index}_{L C P}(A, \Phi)=\operatorname{index}\left(\operatorname{Id}-\mathcal{P}\left(\Lambda^{2}\right)\right) \Phi \mathcal{P}\left(A^{1}\right)=\operatorname{index} A+\operatorname{index} \mathcal{P}\left(\Lambda^{1}\right) \Phi \mathcal{P}\left(A^{1}\right) \text {. }
$$


Instead of the index of the generalized Toeplitz operator $\mathcal{P}\left(A^{1}\right) \Phi \mathcal{P}\left(A^{1}\right)$ we can calculate the index of the elliptic pseudo-differential operator $\left(\mathrm{Id}-\mathcal{P}\left(A^{1}\right)\right)-\Phi \mathcal{P}\left(A^{1}\right)$ of order 0 over $Y$ applying the Atiyah-Singer index theorem. Or we determine the spectral flow $s\left\{\left\{B_{u}\right\}\right.$ of any smooth family of elliptic self-adjoint operators connecting the Dirac operator $\dot{B}_{0}$ with its gauge transform $\Phi^{-1} B_{0} \Phi$. Recall that the spectral flow is the difference between the number of eigenvalues, which change the sign from - to + as $u$ goes from 0 to 1 , and the number of eigenvalues, which change the sign from + to - . It can be described as the index of the suspension $\left\{-\frac{\partial}{\partial u}+B_{u}\right\}$ which is an elliptic operator over $Y \times S^{\mathbf{l}}$.

It is worth mentioning that for Dirac operators there is a one-one correspondence between the linear conjugation problems and the cutting and pasting of Dirac operators; and we obtain the same list of 'correcting' operators and explicit 'error terms' as above. By the decomposition of manifolds and operators into 'elementary' pieces, the cutting and pasting procedure also provides a direct inductive proof of the Atiyah-Singer index theorem for elliptic pseudo-differential operators on closed manifolds.

We close our discussion of the even-dimensional case with a non-additivity theorem. Consider two Dirac operators $A^{j}$ over $X_{j}, j=1,2$, and assume that $A^{1}$ and $A^{2}$ are consistent with regard to Clifford multiplication, i.e. if $A^{1}$ takes the form $G\left(\partial_{u}+B\right)$ close to $Y$, then $A^{2}$ takes the form $G^{-1}\left(\partial_{u}-G B G^{-1}\right)$ close to $Y$, where $u$ denotes the inward normal on $X_{1}$ and $v$ the inward normal on $X_{2}$. Then a Dirac operator $A^{1} \cup A^{2}$ is well defined over $M$ and we obtain as a corollary to Theorem 8:

Theorem 11 Let $E_{\lambda}$ denote the eigenspace of the boundary Dirac operator $B_{0}$ to the eigenvalue $\lambda$, and $P_{\geq \lambda}$ the spectral projection onto the direct sum of all $E_{\alpha}$ with $\alpha \geq \lambda$. Then

$$
\text { index } A^{1} \cup A^{2}=\operatorname{index}\left(A^{1}\right)_{P_{Z \lambda}^{1}}+\operatorname{index}\left(A^{2}\right)_{P_{Z-2}^{2}}+\operatorname{dim} E_{\lambda} .
$$

Note. For $\lambda=0$ we obtain

$$
\text { index }\left(A^{1} \cup A^{2}\right)=\operatorname{index}\left(A^{1}\right)_{P_{Z}}+\operatorname{index}\left(A^{2}\right)_{G \cdot\left(I d-P_{Z}\right) G}+\operatorname{dim} k e r B,
$$

which corresponds exactly to the Novikov additivity of the signature since $\operatorname{sign} X=$ index $A_{\mathrm{P}_{2}}^{+}+\frac{1}{2} \operatorname{dim}$ ker $B$.

\section{Odd Dimension}

If $n$ is odd, the total Dirac operator takes the form $A=G\left(\partial_{u}+B\right)$ near $Y$. Since $G$ is a unitary bundle automorphism with $G^{2}=-\mathrm{Id}_{\left.S\right|_{\gamma}}$, it defines a decomposition of $\left.S\right|_{Y}$ into the direct sum $S^{+} \oplus S^{-}$of the subbundles of the \pm i-eigenvalues of $\left\{G_{y}\right\}_{y \in Y}$. With respect to this decomposition the operator $A$ takes the following form near $Y$ :

$$
A=\left(\begin{array}{cc}
i & 0 \\
0 & -i
\end{array}\right)\left(\partial_{u}+\left(\begin{array}{cc}
0 & B^{-}=\left(B^{+}\right)^{\bullet} \\
B^{+} & 0
\end{array}\right)\right) \text {. }
$$

Notice that the formal self-adjointness of $A=G\left(\partial_{u}+B\right)$ implies $G B=-B G$.

The product form (13) has various far reaching consequences. We begin with the classical cobordism theorem: 
Theorem 12 (Atiyah, Singer [4]) The index of a Dirac operator

$$
B^{+}: C^{\infty}\left(Y ; S^{+}\right) \rightarrow C^{\infty}\left(Y ; S^{-}\right)
$$

over a closed even-dimensional manifold $Y$ vanishes, if the couple $\left(Y, S^{+}\right)$is a 'boundary', i.e. if there exists a manifold $X$ with boundary $Y$ and a bindle of Clifford modules over $X$ which, restricted to $Y$, is equal to $S^{+} \oplus S^{-}$.

Proof From (10) we get for any $A$ of the form (13) close to the boundary $Y$ of an odd-dimensional manifold $X$ index $A_{n_{-}}-$index $A_{\Pi_{+}}=$index $B^{+}$, where $\Pi_{ \pm}$denotes the local elliptic boundary conditions introduced in Example 5. But index $A_{n_{ \pm}}$ vanishes by Green's formula (5).

It follows that $\operatorname{dim}$ ker $B=2 \operatorname{dim} k e r B^{+}$. Moreover, the spectrum of $B$ is $8 y m$ metric with respect to 0 ; hence $\eta_{B}(z) \equiv 0$; and the index density $\alpha$ vanishes for odd-dimensional $X$. This reduces Theorem 8 to the simple formulas

$$
\begin{aligned}
\text { index } A_{\mathrm{P}_{Z}(B)} & =-\operatorname{dimker} B_{+}=\mathrm{i}\left(\mathrm{P}_{\geq}(B), \mathcal{P}_{+}(A)\right) \quad \text { and } \\
\text { index } A_{R} & =-\operatorname{dimker} B_{+}+\mathrm{i}\left(R, \mathrm{P}_{\geq}(B)\right) .
\end{aligned}
$$

That a kernel dimension appears indicates the non-homotopy invariance, see the example in Hitchin [21].

Now we discuss the true odd situation, i.e. we are given an odd-dimensional closed Riemannian manifold $M$ which is partitioned in two manifolds $X_{1}, X_{2}$ will boundary by a hypersurface $Y$, and a bundle $S$ of Clifford modules over $M$. We fix a bicollar neighbourhood $N$ of $Y$ in $M$. We assume that the metrics of $M$ and $S$ are product near $Y$.

The invariants we study on odd-dimensional manifolds are spectral invariants: the spectral now, the $\eta$-invariant, and the analytic torsion. They are defined by the spectra of self-adjoint Dirac operators over $M$ or $Y$; by the spectra of self-adjoint boundary problems; by the spectra of associated Laplacians; or by the spectra of naturally associated families. We shall begin with the spectral fow introduced above after Theorem 12. It plays a prominent role in the recent work of 'three-dimensional' topologists where it is used to determine the value of Casson's invariant and other invariants introduced recently (see Taubes [37], Witten [38]).

Let $\mathcal{D}$ denote the space of cylindrical Dirac operators acting on $S$ with fixed principal symbol $G(x, \xi)$. Cylindrical means that the operator has the form (13) in the fixed cylinder $N$; i.e. elements of $D$ differ over $N$ only by endomorphisms of the bundle $S$. Let $D^{\times}$denote the subspace of invertible Dirac operators. We consider families

$$
\mathcal{A}=\left\{A_{r}\right\}:(I ;\{0,1\}) \rightarrow\left(\mathcal{D} ; \mathcal{D}^{x}\right) .
$$

It is not difficult to see that $s f\left\{A_{r}\right\}$ is an integer-valued homotopy invariant of such families over $M$. In order to obtain an odd variant of Bojarski's theorem we need an invariant of families defined over $Y$. This was worked out recently by $L$. Nicolaescu [31]. Once again, the key is the 'twisted' orthogonality of Cauchy data of (8) which in this case says

$$
\mathcal{P}(\Lambda)=-G\left(\mathrm{Id}-\mathcal{P}\left(\Lambda^{*}\right)\right) G=-G(\mathrm{Id}-\mathcal{P}(\Lambda)) G .
$$


This equation has the following nice interpretation. Recall that $\left.G\right|_{Y}$ defines a symplectic structure on $L^{2}\left(Y ;\left.S\right|_{Y}\right)$

$$
\left\{s_{1}, s_{2}\right\}:=\left(G s_{1}, s_{2}\right)=\int_{Y}\left\langle G s_{1}(y) ; s_{2}(y)\right\rangle d y .
$$

Theorem 13 ([9]) For $A \in D$, the Cauchy dato space $H\left(A^{1}\right)$ is a Lagranginn subspace of $L^{2}\left(Y ;\left.S\right|_{Y}\right)$ with respect to $G$.

Here $H\left(A^{1}\right)$ denotes the Cauchy data space of the operator $A^{1}:=A \mid x_{1}$. Lagrangian means that $G\left(H\left(A^{2}\right)\right)$ and $H\left(A^{2}\right)$ are orthogonal to each other and span the whole space. It is an easy consequence of Kuiper's theorem that the space of all Lagrangian subspaces is contractible (in the operator topology of the corresponding projections). To get a situation which is interesting from a topological point of view we consider the space $\mathcal{F}_{2}^{G}$ of all Fredliolm pairs of Lagrangian subspaces of $L^{2}\left(\left.Y_{;} S\right|_{Y}\right)$. We have the following result proved by Nicolaescu [31] (for the definition of the $K$-group $K R^{-7}$ see Karoubi [22]):

Theorem 14 (Nicolaescu [31]) The space $\mathcal{F}_{2}^{G}$ has the homotopy type of a classifying space for $K R^{-7}$; in particular

$$
\pi_{1}\left(\mathcal{F}_{2}^{G}\right) \simeq \mathbf{Z}
$$

It follows that to any loop $\left\{H_{r}^{1}, H_{r}^{2}\right\}$ of elements of $\mathcal{F}_{2}^{G}$ corresponds an integer $\mu\left\{H_{r}^{1}, H_{r}^{2}\right\}$, which is an obvious generalization of the standard Maslov index defined in finite dimensions (see Arnold [2]). Following standard procedures we can define the Maslov index for any path in $\mathcal{F}_{2}^{G}$ and formulate the 'odd' variant of Bojarski's theorem.

Theorem 15 (Nicolaescu $[31]$ ) Let $\left\{A_{r}\right\}:(I ;\{0,1\}) \rightarrow\left(\mathcal{D} ; D^{\times}\right)$be a smooth family of cylindrical Dirac operators on $M$ acting on $S$. Then

$$
\text { sf }\left\{A_{r}\right\}=\mu\left\{H\left(A_{r}^{1}\right), H\left(A_{r}^{2}\right)\right\} .
$$

This theorem leads to a decomposition formula for the spectral fow. Let $\mathcal{L}_{1}, \mathcal{L}_{2}$ denote subspaces of ker $B$ such that range $\left(\mathrm{P}_{>}\right)+\mathcal{L}_{j}$ are Lagrangian subspaces of $L^{2}\left(Y ;\left.S\right|_{Y}\right.$ ) (or, equivalently, $\mathcal{L}_{j}$ is a Lagrangian subspace of $\operatorname{ker} B$ which is a finitedimensional symplectic space). We denote by $A_{\mathcal{L}_{j}}^{j}, j=1,2$ the operator

$$
\left\{\begin{array}{c}
A_{C_{j}}^{j}=A^{j}:=\left.A\right|_{X_{j}} \\
\operatorname{dom} A_{\mathcal{C}_{j}}^{j}:=\left\{s \in \mathcal{H}^{1}\left(X_{j} ;\left.S\right|_{X_{j}}\right) \mid\left(\mathrm{P}_{>}+\pi_{j}\right)\left(\left.s\right|_{Y}\right)=0\right\},
\end{array}\right.
$$

where $\pi_{j}$ denotes the projection onto $\mathcal{L}_{j}$. Then $A_{\mathcal{L}_{j}}^{j}$ is a closed sclf-adjoint operator in $L^{2}\left(X_{j} ;\left.S\right|_{X_{j}}\right)$. Varying $A$ (and so also its 'tangential' Dirac operator $B$ ) and choosing families $\left\{\mathcal{L}_{j, r}\right\}$, the following result follows from the work of Nicolaescu.

Theorem $16 \operatorname{sf}\left\{A_{r}\right\}=\operatorname{si}\left\{\left(A_{r}^{1}\right)_{\mathcal{L}_{1, r}}\right\}+\operatorname{sf}\left\{\left(A_{r}^{2}\right)_{\mathcal{C}_{2, r}}\right\}+\mu\left\{\mathcal{L}_{1, r}, \mathcal{L}_{2, r}\right\}$. 
This theorem has already been used in the work of Kirk and Klassen [23] in their computation of topological invariants of 3-manifolds.

We have a similar result for the $\eta$-invariant. The operator $A_{c}^{1}$ has a discrete spectrum and the $\eta$-invariant of such operators is well defined (see [15]). The next result follows from the work of Lesch and Wojciechowski [27] and an observation made by $W$. Müller [30] that, modulo the integers, the $\eta$-invariant on manifolds of the form $M=X_{1} \cup[-R, R] \times Y \cup X_{2}$ with cylindrical $A$ does not depend on the length $R$ of the cylinder.

Theorem 17 Let $\eta\left(\mathcal{L}_{1}, \mathcal{L}_{2}\right)$ denote the $\eta$-invariant of $A$ on the cylinder $[-1,1] \times Y$ with boundary condition $\mathcal{L}_{1}$ at $u=-1$ and $\mathcal{L}_{2}$ at $u=1$. Then we have

$$
\eta(A) \equiv \eta\left(\left(A^{1}\right) \mathcal{C}_{2}\right)+\eta\left(\left(A^{2}\right) \mathcal{C}_{2}\right)+\eta\left(\mathcal{L}_{1}, \mathcal{L}_{2}\right) \bmod \mathbf{Z}_{1}
$$

Note. The integer contribution has been computed recently by U. Bunke [13].

\section{History and Perspectives}

For learning the basic relations between Dirac operators and global analysis on manifolds without boundary we refer to Palais [32], Karoubi [22], Gilkey [16], [17], Lawson and Michelsohn [26], and Berline, Getzler, and Vergne [5]; for details of the calculus on manifolds with boundary as sketched in the sections 1-4 of this article see Booß and Wojciechowski [11]; for other approaches than presented here in Section 5 to the 'odd' problem and to the cutting and pasting of $\eta$-invariants and analytic torsion over partitioned manifolds see Gilkey and Smith [19], [20], Roe [33], Singer [36], Booß and Wojciechowski [10], Gilkey [18], Mazzeo and Melrose [29], Klimek and Wojciechowski [24], [25], and Lück [28].

\section{References}

1. Agranoviz, M.S., and Dynin, A.S.: 1962, 'General boundary value problems for elliptic systems in an n-dimensional domain', Dokl. Aked. Neuk SSSR 140, 511.511. (Russian; Engliah translation Soviet Math. Dokl. 3 (1962/63), 1323-1327).

2. Amold, V.I.: 1967, 'Characteristic class entering in quantizntion conditions', Funkcional. Anal. i Prilozen. 1, 1.14. (Russian; English translation Functional Anal. A ppl. 1, 1-13; French translation Complément 1 to V.P. Maslov, Théorie des Perturbations et Méthodes Asympto. tipues. Dunod, Gauthier-Villars, Paris 1972, 341-361).

3. Atiyah, M.F., Patodi, V.K., and Singer, I.M.: 1975, 'Spectral anymmetry and Riemannian geometry. I', Math. Proc. Cembridge Phil. Soc. 77, 43-69.

4. Atiyah, M.F., and Singer, I.M.: 1963, 'The index of elliptic operators on compact manifolds', Bull. Amer. Moth. Soc. 69, $422-433$.

5. Berline, N., Getzler, E., and Vergne, M.: 1992, Heet Kernels and Dirac Operators, Springer, Berlin.

6. Birman, M., and Solomyak, A.: 1982, 'On subspaces which admit pseudodifferentinl projecLions', Vesinik Leningrad Univ. Nat. Mekh. Astronom. 82, no. 1, 18-25 (Russian).

7. Bojarski, B.: 1979, 'The abstract linear conjugation problem and Fredlinlin pairs of subspaces', in: In Memoricm I.N.Vekua Tbilisi Univ., Tbilisi, pp. 45-60 (Russinn).

8. BooB, B., and Wojciechowski, K.P.: 1985, 'Desuspension of splitting elliptic symbols I', Ann. Global Anal. Geom. 3, 337.383. 9. $-1,-$ : 1986, 'Desuspension of splitting elliptic symbols II', Ann. Global Anal. Geom. 4 ,
349-400. 
10. - - : 1989, 'Pacudo-difterential projectione and the topology of certain spacen of elliptic boundary value problems', Comm. Math. Phyo. $121,1.9$.

11. -, -: 1893, Elliplic Boundery Problems for Dirac Operelore, Birkhn̈uner, Boston (in print).

12. Branson, Th.P., and Gilkey, P.B.: 1992, 'Residues of the eta function for an operator of Dirac type', J. Funct. Anal. 108, 17-87.

13. Bunke, U.: 1993, A Glueing Formula for the ela-Invariant, Preprint, Humboldt Univernilat Berlin.

14. Calderón, A.P.: 1963, 'Boundary value problems for elliptic equations', in: Outlines of the Joint Soviet-American Symposixm on Partial Differential Equations, Novosibirak, Pp. $303-$ 304.

15. Douglas, R.G., and Wojciechowaki, K.P.: 1991, 'Adinbatic limils of the $\eta$-invariants. The odd-dimensional Atiynh-Palodj-Singer problem', Comm. Meth. Phyo. 142, 139-168.

16. Gilkey, P.B.: 1984, Invarience Theory, the Heat Equation, and the Atiyah-Singer Index The. orem, Publish or Perish, Wilmington. New revised edition in preparation.

17. -: 1989, 'The geometrical index theorem for Clifford modules', in: Rassias, T.M. (ed.), Topica in Mothemetical Analyois, World Scientific Press, Singapore, pp. 31 5-327.

18. - : 1991, On the Index of Geometrical Operators for Riemannien Menifolde with Bosndery, Preprint, University of Oregon, Eugene.

19. Gilkey, P.B., and Smith, L.: 1983n, 'The eta invariant for a clasn of clliptic boundary value problems', Comm. Pure Appl. Math. 36, 85-131.

20. $-1,1983 \mathrm{~b}$, 'The twisted index problem for manifolds with boundary', J. Diflerential Geom. 18, 393-444.

21. Hitchin, N.: 1974, 'Ilarmonic spinors', Adv. Math. 14, 1-35.

22. Karoubi, M.: 1878, K. Theory, Springer, Berlin.

23. Kirk, P.A., and Klassen, E.P.: 1993, Computing Spectral Flow vie Cup Produets, Preprint, Indiana University, Bloomington.

24. Klimek, S., and Wojciechowski, K.P.: 1992, Adiabatic Cobordism Theorems for Analytic Torsion and $\eta$-Invariant, Preprint, Purdue University, IUPUI, Indiannaolis.

25. -, -: 1993, ' $\eta$-Ivarinnts on manifolds with cylindrical end', DiDer. Geom. Appl. 3 (1993), to appear.

26. Lawson, H.B., and Michelsolin, M.-L.: 1989, Spin Geometry, Princelon University Press, Princeton.

27. Lesch, M., and Wojciccliowski, K.P.: 1003, On the $\eta$-inveriant of generalized Aliyah-PatodiSinger boundary value problems, Report No. 278, Universitäl Aligsburg.

28. Lück, $W .:$ 1993, 'Analytic and topological torsion for manifolds with boundary and symmetry', J. Differential Geom. 37, 263-322.

29. Mazzeo, R.R, and Melrose, R.B.: 1992, Analytic Surgery and the Ele Invariant, Preprint, M.I.T.

30. Müller, W.: 1993, Eta-Invariants and Manifolds with Boundary, Preprint, Max-PInnck-Inst. f. Math., Bonn.

31. Nicolaescu, L.: 1993, The Maslov Index, the Spectral Flow, and Splitfings of Manifolda, Preprint, Michigan State University, East Lansing.

32. Palais, R.S. (ed.): 1965, Seminer on the Atiyah-Singer Index Theorem, Ann. of Math. Studien 57, Princeton University Press, Princeton.

33. Roe, J.: 1988, 'Partitioning non-compact manifolds and the dual Toeplitz problem', in: $O p$ eretor algebres and epplication. Vol. 1, Lond. Math. Soc. Lect. Note Ser. 135, pp. 187-228.

34. Seeley, R.T.: 1966, 'Singular integrals and boundary value problems', Amer. J. Math. 88, 781.809.

35. -: 1969, 'Topics in pseudo-differential operators', in: CIMIE Conference on PoexdoDifferential Operators (Strese 1968). Ed. Cremonese, Rome, 1069, pp. 167.305.

36. Singer, I.M.: 1988, 'The $\eta$-invariant and the index', in: Yall, S.-T. (cd.), Malhematical Aspects of String Theory, World Scientific Press, Singapore, pp. 239-258.

37. Taubes, C.H.: 1990, 'Casson's invariant and gauge theory', J. Differential Geom. 31, 547.599.

38. Witten, E.: 1989, 'Quantum field theory and the Jones polynomial', Comm. Math. Phys. 121, 351-400.

39. Wojciechowski, K.P.: 1985, 'Elliptic opernlore and relative $K$-homology groups on manifolds with boundary', C.R. Math. Rep. Acad. Sci. Canada 7, 149-151. 
Liote over tidligere udkorme tekster tilaendes gerne. Benvendeloe heram kan oke til MTUFA'B oekretariat tlf. $167577 \mathrm{Il}$ lokal 2863
227/92 "Computersimulering og fysik" af: Per M.hansen, Steffen Holm, Peter Maibom, Mads K. Dall Petersen, Pernille Postgaard, Thomas B.Schroder, Ivar P. Zeck

Vejleder: Peder Voetmann Christiansen

$228 / 92$ "Teknologi og historie" Fire artikler af:

Mogens Nis8, Jens Hoyrup, Ib Thiersen. Hans Hedal

$229 / 92$ "Masser af Information uden betydning" En diskussion af informationsteorien $i$ Tor Nortetranders" "Mrrk Verden" og en skitse til et alternativ basseret pl andenordens kybernetik og semiotik. af : soren Brier

217/92 "Two papers on APPLICATIONS AṂ WODELLING IN THE MATHEMATICS CURRICULIM"

by: Mogens M1es

218/92 "A Three-Square Theorem"

by: Lars Kadison

219/92 "RUPNOK - Etationer straming 1 elastiske ror" af: Anja Boisen, Karen Birkelund, Mette Olufsen Vejleder: Jesper Larsen

220/92 "Automatisk diamosticering 1 digitale kredslob" af: Bjorn Christensen, Ole Moller Nielsen Vejleder: Stig Andur Pedersen

221/92 "A BUNDLE VALUED RADON TRANSFORM, WITH APPLICATIONS TO INVARIANT WAVE EQUATIONS"

by: Thomas P. Branson, Gestur Olafsson and Henrik Schlichtkrull

222/92 On the Representations or some Infinite Dimensional. Groups and Algebras Related to Guantum Physics

by: Johnny $T$. Ottesen

223/92 THE FUNCTIONAL DETERNINANT

by: Thomas P. Branson

224/92 UNTVERSAL AC CONDUCTIVITY OF MON-METALLTC SOLTDS AT, LOW TEMPERATURES

by: deppe C. Dyre

225/82 "BATNODELLEN" Impodans8pektroskopi i ultrarent en-kryotallinok oilicivon

af: Anja Boisen, Andere Gorm Larsen, Jesper Varmer, Johannes $X$. Bieloen, Kit R. Bemsen, Peter Beggild og Thomas Bougaard

Vejleder: Petr Viscor

226/92 METBODS AWD MODELS FOR ESTINATING THE GLOBAL CIRCULATION OF SELECTED EMISSIONS FROM ENERGY CONVERSION"

by: Bent Sorensen $230 / 92$ "Vinklens tredeling - et klassisk
problem"

et matematisk projekt af

Raren Birkelund, Bjozn Christensen

Vejleder: Johnny Ottesen

$231 \mathrm{~A} / 92$ "Elektrondiffusion i sillcium - en matematisk model"

af: Jesper Voetmann, Karen Birkelund. Mette Olufien, Ole Moller Nielsen

Vejledere: Johnny Ottesen, H.B.Hansen

2318/92 "Elektrondiffusion i silicium - en matematisk model" Rildetekster

af: Jesper Voetmann, Raren Birkelund, Mette Olufsen, Ole Moller Nielsen

Vejledere: Johnny Ottesen, H.B.Hansen

232/92 "Undersogelse om den simultane opdagelse af energiens bevarelse og iserdeles om de af Mayer. Colding, Joule og Helmholtz uaforte arbejder"

af: L.Arleth, G.I.Dybkjer, M.T.Ostergåra Vejleder: Dorthe Posselt

233/92 The effect of age-dependent host mortality on the dynamics of an endemic disease and Instability in an sIR-model with agedependent susceptibility

by: Viggo Andreasen

234/92 "THE FUNCTIONAL DETERMIMANT OF A FOUR-DIMENSIONAL BOUNDARY VALUE PROBLEM"

by: Thomas P. Branson and Peter B. Gilkey

235/92 OVERFLADESTRUKTUR OG POREUDVIKLING AF KOKS

- Modul 3 rysik projekt -

af: Thomas Jessen 
236a/93 INTRODURTION TIL KVANTE HALL EFFEKTEN

ar: Anfo Boisen, Peter Boggild

Vejleder: Peder Voetmann Chrietiansen Erland Brun Hansen

236b/93 STROMSSAMMENBRUD AF RVANTE HALL EFFEKTEN

ar: Anje Bolsen, Peter Begeild

Vejleder: Peder Voetsenn Christianen Erland Brun Hansen

$237 / 93$ The vedderburn princlpel theorem and Shukla cohomology

ef: Lare kadison

$238 / 93$ SENIOTIK OG SYSTENEGENSKABER (2)

Vektorbind og teneorer

ef: Peder Voeteann chrietianeen

$239 / 93$ Valgsystemer - Modelbygning og analyse

Matemat1k 2 . modul

ar: Charlotte Gjerrild, Jane Hansen.

Maria Hermannsion, Allan Jorgensen.

Ragna Cleuson-Rass. Poul Lützen

Vejleder: Mogens Nise

240/93 Patologiske eksempler.

On ene matematioke risks betydning for den matematiake udvikling

ar: Claus Dreby, Jorn Skov Hansen, Run: Ulsoe Johansen, Peter melbom, Johannes Rrietofrer Nielsen

Vejleder: Mogens NisB

241/93 FOTOVOLTAISK STATUSNOTAT 1

af: Bent Sorensen

$242 / 93$ Brovedligeholdelse - bevar mig vel

Analyse af Vejdirektoratets model for optimering af broreparationer

af: Iinda Kyndlev, Kare Pundal, Kama

Tulinius, Ivar zeck

Vejleder: Jesper Larsen

243/93 TANREERSPERIMENTER I FYSIRKEN

Et 1 .modul fysikprojekt

af: Karen Birkelund, stine Sofia Korremann

Vejleder: Dorthe Posselt

244/93 RADONTRANSFORMATIONEN oq dens anvendelse

1 CT-scanning

Projektrapport

af: Trine Andreasen, Tine Guldager Christiansen,

Nina Skov Hansen og Christine Iversen

Vejledere: Gestur olafseon og Jesper Larsen

$245 a+b$

Time-of-Flight malinger p\& krystaliinske

halvledere

Specialerapport

af: Linda Szkotak Jensen og Lise Odgaard Gade

Vejledere: Petr Viscor og Niels Boye Olsen

$246 / 93$ HVERDAGSVIDEN OG MATEMATIK

- LEREPROCESSER I SKOLEN

af: Lena Lindenskov, Statens Humanistiske

Forskningsrad, RUC, IMFUFA
247/93 UNIVERSAL LOW TEMPERATURE AC CONDUCTIVITY OF MACROSCOPICALLY DISORDERED NON-METALS

by: Jeppe C. Dyre

248/93 DIRAC OPERATORS AND MANIFOLDS WITH BOUNDARY

by: B. Booss-Bavnbek, K.P.Wojciechowski

$249 / 93$ Perspectives on Teichmüller and the Jahresbericht Addendum to Schappacher. scholz, et al.

by: B. Booss-Bavnbek

With comments by W.Abikoff. L.Ahlfors, J.Cerf, P.J.Davis, W.Puchs, F.P.Gardiner, J.Jost, J.-P.Rahane, R.Lohan, L.Lorch, I.Radkau and T.Söderquist

250/93 EULER OQ BOLZANO - MATEMATIST ATALISE SET I ET VIDENSKABSTEORETJSK PERSTEKTIV

Projektrepport af: Anja Juul, Lone Nichelsen, Tomas Bojosind Jensen

Vejleder: Stig Andur Pedersen 